\section{Keine Pille für den Mann in Sicht}

Die „Pille für den Mann“ - sie wird es so schnell nicht geben. Die umständliche Applikation per Spritze und ein hoher Anteil von Nonrespondern machen der Forschung zu schaffen. Bislang hat noch kein einziges pharmakologisches Kontrazeptivum zur Anwendung beim Mann den Durchbruch geschafft. Dies liegt weniger daran, dass eine solche Kontrazeption nicht möglich wäre: Nebenwirkungen, eine umständliche Anwendung, häufige Kontrollen und die lange Zeit bis zur wirksamen Unterdrückung der Spermatogenese sind eher Gründe für das Scheitern der bisherigen Ansätze, berichten Mediziner um Dr. Mara Roth, Seattle, in einer Übersichtsarbeit.

Roth MY et al. Andrology 2015 (online first)

\section{PCA3 soll PSA-Test sicherer machen}

Das National Cancer Institute der USA validierte in einer Studie mit 859 Teilnehmern die diagnostische Wertigkeit des Prostatakrebs-Antigens 3 (PCA3) im Urin, die beim Screening durch einen erhöhten PSA-Wert oder weniger als 15\% freies PSA aufgefallen waren und nun biopsiert werden sollten. Ein Wert $>60$ wurde als hohes, eine Wert $<20$ als niedriges Risiko gewertet. Der positive prädiktive Wert eines PCA3-Werts $>60$ lag bei erstmaliger Biopsie bei $80 \%$, der negative prädiktive Wert $<20$ sogar bei $88 \%$. Damit könnte dieser Tumormarker helfen, Patienten mit Anfangsverdacht im PSA-Test zu identifizieren, die ein hohes Risiko für ein Prostatakarzinom haben.

Wei JT et al. J Clin Oncol 2014; 32(36): 4066-4072

\section{Alt, dement \& inkontinent?}

Besonders für Betagte kann eine Blasenschwäche fatale Folgen haben, denn Inkontinenz und Multimorbidität verstärken sich im Alter oft gegenseitig. Kommt noch eine Demenz hinzu, gestaltet sich eine effektive Therapie als echte Herausforderung. Urologen raten daher, z. B. im Rahmen eines Hausbesuchs herauszufinden, inwieweit sich das Umfeld des Patienten verbessern lässt und wie Sie die Therapie optimal anpassen. Näheres erfahren Sie im Schwerpunkt „Urologie“.

Claudia Daniels Redakteurin

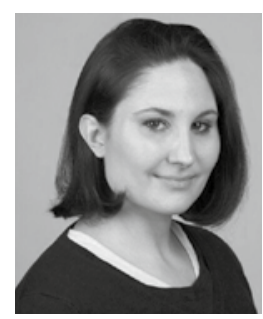

\title{
Therapieoptionen bei BPS erweitert
}

Für die Behandlung von Patienten mit benignem Prostatasyndrom (BPS) steht inzwischen eine große Palette konservativer, operativer und interventioneller Verfahren zur Verfügung. Nicht alles ist bereits in der BPS-Leitlinie berücksichtigt. Medikamentöse Kombinationen verschiedener Wirkmechanismen sind effektiver als die jeweiligen Einzelsubstanzen. Eine neue und noch nicht in den Leitlinien enthaltene Option ist die Kombination eines PDE-5-Inhibitors mit einem 5alpha-Reduktasehemmer. So habe sich in einem Direktvergleich der Kombination Tadalafil/Finasterid mit Finasterid/Placebo eine signifikante Verbesserung beim Symptomenscore IPSS zugunsten der Kombination ergeben, so Prof. Dr. Rolf Muschter, Rotenburg. Therapeutische Neuigkeiten auf dem Gebiet der BPS sind vor allem instrumenteller Art, was Destruktions-, Ablations- und Retraktionstechniken angeht, aber auch die von interventionellen Radiologen praktizierte Embolisation von Prostataarterien.

Kongresszeitung zum 67. Kongress der DGU 2015

\section{Erste deutsche S3-Leitlinie zum Nierenzellkarzinom}

Beim fortgeschrittenen oder metastasierten klarzelligen Nierenzellkarzinom hat die zielgerichtete Therapie inzwischen ihren festen Platz. In der ersten deutschen, durch die Fachgesellschaften getragenen S3-Leitlinie zu Diagnostik und Therapie erhält dieses Vorgehen den höchsten Empfehlungsgrad. Es haben sich beispielsweise Präparate der zielgerichteten Therapie durchgesetzt, die zur Gruppe der VEGFHemmer oder der mTOR-Inhibitoren gehören. Bei Patienten, die ein fortgeschrit- tenes und/oder ein metastasiertes klarzelliges Nierenzellkarzinom mit niedrigem oder intermediärem Risiko haben, sollen der Leitlinie zufolge in der Erstlinientherapie Sunitinib, Pazopanib oder Bevacizumab plus IFN verwendet werden. In der Zweitlinientherapie nach einer Behandlung mit Sunitinib oder mit Zytokinen soll Axitinib verwendet werden.

Leitlinie Nierenzellkarzinom - Langversion 1.0 September 2015. AWMF-Registernummer: 043/017-OL

\section{Sono des Harntrakts bei Endometriose!}

Ziel einer prospektiven Beobachtungsstudie war es, zu überprüfen, ob eine routinemäßige Darstellung von Blase, Ureter und Nieren mit transvaginalem Ultraschall bei Frauen mit Verdacht auf Endometriose sinnvoll ist, um eine Beteiligung der Harnwege bzw. eine bestehende Hydronephrose möglichst frühzeitig zu erkennen. In der 14-monatigen Studie wurde bei 335 von 848 Patientinnen (39,5\%) per Ultraschall eine Endometriose diagnostiziert. Bei 17 Frauen (2\%) war im ersten Ultraschall die Endometriose im Harntrakt erkennbar. 14 Patientinnen hatten einen Ureterbefall, bei drei Frauen waren Blase und Ureter beteiligt und bei drei weiteren nur die Blase. 1,3\% zeigten im Ultraschall andere Anomalien des Harntrakts. Bei 12 der 17 Patientinnen mit Harntrakt-Endometriose bestanden Hinweise auf eine Hydronephrose. Bei allen operierten oder mit CT bzw. MRT weiteruntersuchten Frauen wurde die Ultraschall-Diagnose des Ureter- und/oder Blasenbefalls bestätigt. Die Autoren plädieren deshalb dafür, die Untersuchung des gesamten Harntrakts in die Ultraschalldiagnostik bei Frauen mit Verdacht auf Endometriose zu integrieren.

Pateman Ket al. Hum Reprod 2015 (online first) 